\title{
BEDEUTUNG DER MOLEKULARBIOLOGIE FÜR DIE DIAGNOSTIK UND PROGNOSE KOLOREKTALER KARZINOME IN DER CHIRURGISCHEN PRAXIS
}

\author{
Michael Dlouhýa, Miloslav Duda ${ }^{a}$, David Kalvoda ${ }^{a}$ und Petr Beneš ${ }^{b}$ \\ a II. Chirurgische Universitätsklinik der Palacký-Universität, Olomouc, Tschechische Republik \\ b Onkologische Universitätsklinik der Palacký-Universität, Olomouc, Tschechische Republik
}

Eingegangen am 23. September 2000

Key words: Molecular biology / Colorectal cancer / Prognosis

Using the recently developed techniques of molecular biology, staging and follow up of patients with malignant diseases will improve. At the University Hospital in Olomouc, patients suffering from colorectal cancer are registred and a intensive prospective follow up is documented. In addition, a variety of parameters measured with methods using molecular biology is used to describe further the disease of the individual patient. Combining these data, it is hoped, that staging and monitoring of adjuvant treatment is improved.

\section{EINLEITUNG}

Der Fortschritt in der Onkologie verschiebt sich heute vom Bereich modernster präklinischen Diagnostik z. B. durch Computertomographie auf die Ebene der einzelnen Tumorzellen, wo durch molekularbiologische Methoden neue Erkenntnisse gewonnen werden können. Ergebnisse dieser Forschungen von Tumorerkrankungen auf die Ebene der Einzelzellen werden nach heutigen Erkenntnissen in näherer Zukunft eine wichtige Rolle im Kampf gegen Tumoren spielen. Die Mehrzahl dieser Methoden ist leider sehr teuer und auch noch im Stadium der präklinischen Evaluation und so ist ihre Einführung in die alltägliche klinische Anwendung noch mit Problemen verbunden. Bei der Auseinandersetzung mit dieser Problematik muss man sich auch als überwiegend klinisch tätiger Chirurg sehr gut informieren und sich mit neuen Erkenntnissen vertraut machen und vor dieser Herausvorderung nicht zurückschrecken ${ }^{16,17}$.

Die Analytik des genetischen Materials einer Tumorzelle dient zur Präzisierung der Tumordiagnostik und möglicherweise auch in Zukunft zum Monitoring des Erfolges einer Tumortherapie und zur prognostischen Beurteilung einer Tumorerkrankung. Auf der Analyse von Einzelzellreaktionen beruht auch die sog. Tumorimmunologie ${ }^{2,20}$. Aus diesen beiden Forschungsrichtungen dürften in Zukunft die Hauptimpulse für Therapiestrategien gegen Tumoren erwachsen. Im Augenblick gilt jedoch weiterhin, dass die wesentliche therapeutische Maßnahme eine radikale chirurgische Entfernung des Tumors ist. In zweiter Linie treten dann adjuvante Chemo- und Radiotherapien bzw. jetzt langsam auch zunehmend die sog. Immunotherapie in Aktion. Durch die Untersuchung des Tumorgewebes mit den o. g. molekularbiologischen Methoden verschiebt sich die
Bewertung eines Tumorleidens von der reinen diskretiven morphologischen Betrachtung zu einer mehr funktionell molekularbiologisch bestimmten ${ }^{1,7,10,19}$. Eine wichtige Charakterisierung von Tumorzellen sind quantitative Veränderungen in DNA-Gehalt. Eine hierzu zu verwendende Methode ist die sog. Durchflußzytometrie. Es hat sich herausgestellt, dass z. B. die Zahl vorhandener Chromosomensätze in einer Tumorzelle einen prognostisch relevanten Faktor darstellt, eine solche Untersuchung kann mit der Durchflußzytometrie gut durchgeführt werden. Wir selbst verwenden darüber hinaus noch die Methode des Nachweises bestimmter intrazellulärer Bestandteile der DNA oder intrazellulärer Proteine, die durch verschiedene Fluoreszensfarbstoffe markiert werden können und die ebenfalls relevante Prognoseparameter für das Tumorleiden darstellen $^{3,9,14,15}$.

\section{KONZEPT DES VORGEHENS IN OLOMOUC}

Der gesamte Bereich der Forschung auf zellulärer Ebene entwickelt sich auch bei uns sehr intensiv und ist in rascher Bewegung. Um hierbei die Übersicht zu behalten und Methoden zu entwickeln, die in der klinischen Praxis gut anwendbar sind, ist es erforderlich, ein Schema für das diagnostische Vorgehen aufzustellen ${ }^{5}$, 6, 11, 12. Ziel der molekularbiologischen Untersuchungen des gewonnenen Materials muss die Präzisierung der Tumordiagnostik, das Monitoring der Tumorerkrankung und die Dokumentation der Reaktion auf Antitumortherapien sein, so dass das Ergebnis dieser Forschungen direkt dem Patienten im Sinne einer Therapieoptimierung zu Gute kommt ${ }^{4}, 8,13,18$. Dafür ist es nötig, umfangreiche statistische Erhebungen im Sinne von 
Tumorregistern zu bilden, bei denen möglichst viele Parameter mit der Prognose der Patienten korreliert werden müssen. In dieser Phase befinden wir uns in der Gegenwart in Olomouc und wir standardisieren die durchzuführenden Untersuchungen im Rahmen einer prospektiven Untersuchung.

\section{ERGEBNISSE}

Die Inzidenz des kolorektalen Karzinoms in unserer Population ist sehr hoch ${ }^{21}$. Die Tschechische Republik nimmt in der europäischen Statistik leider einen der ersten Plätze ein. Die Abbildungen 1 und 2 zeigen ein allmähliches Ansteigen der Inzidenz des kolorektalen Karzinoms in Tschechien, im Bezirk Olomouc und in Bezug auf unsere eigene Klinik, die eine der vier chirurgischen Erfassungsstellen in unserem Bezirk ist. In den letzten 10 Jahren haben wir in unserer Klinik insgesamt 253 Kranke an einem kolorektalen Karzinom operiert, bei denen insgesamt 265 radikale und palliative Eingriffe durchgeführt wurden (Tab. 1). Die Lokalisation des kolorektalen Karzinoms in diesem Krankengut entspricht dabei den bereits bekannten statistischen Verteilungen (Tab. 2). Die Ergebnisse unserer chirurgischen Therapie bei diesem Krankengut zeigt Tabelle 3. Tabelle 4 zeigt die klassischen onkologischen Marker mit Spezifität für das kolorektale Karzinom. Die Ergebnisse dreier Parameter zur Beurteilung der Zellenaktivität werden in den Tabellen 5, 6, 7 und 8 angezeigt. Diese Daten geben den hohen Stand der Aktivitäten der Tumorforschung im Bereich der zellulären und subzellulären Strukturen in Olomouc wieder, diese Ergebnisse beruhen auch auf einer internationalen Zusammenarbeit mit verschiedenen Labors.

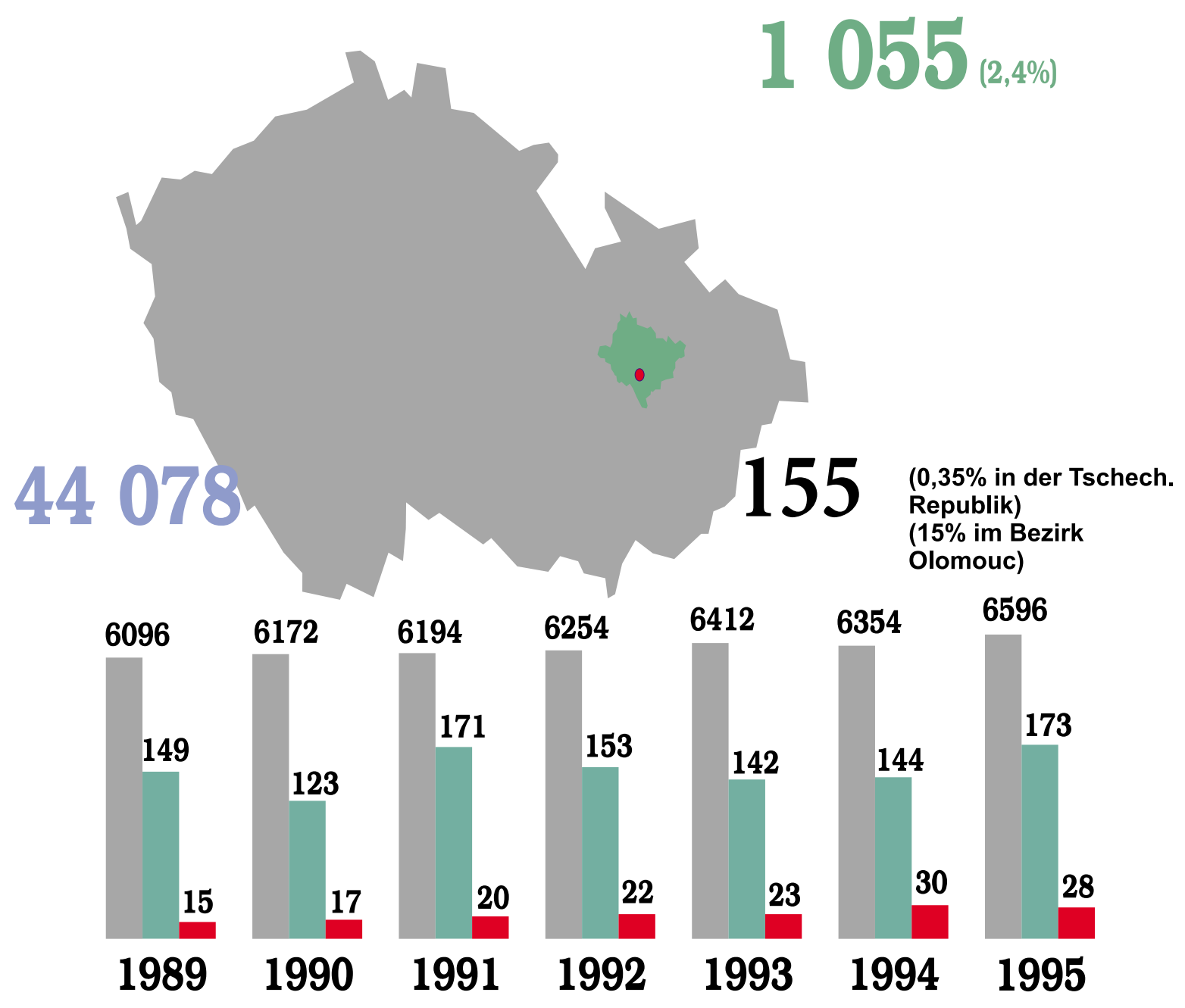

Abb. 1 Inzidenz des kolorektalen Karzinoms in der Tschechischen Republik, im Bezirk Olomouc und auf der II. chirurgischen Klinik 1989-1995 


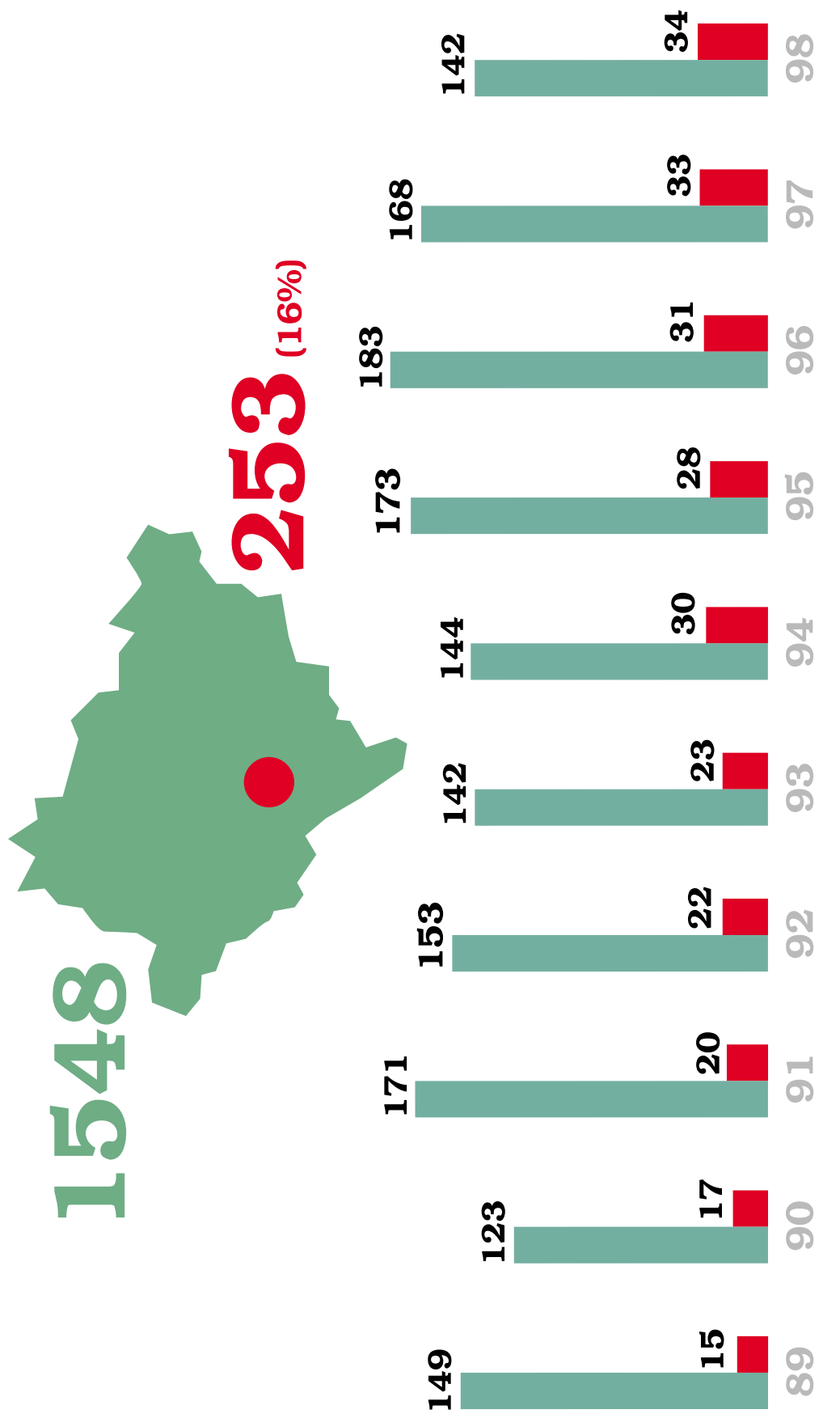

Abb. 2 Inzidenz des kolorektalen Karzinoms im Bezirk Olomouc und auf der II. chirurgischen Klinik 1989-1998 
Tabelle 1. Chirurgie des kolorektalen Karzinoms Eigenes Patientenkollektiv 1989-1998

\begin{tabular}{lc}
\hline Die Zahl der Kranken & 253 (Alter 24-97 J.) \\
Männer & 149 (durchschn. Alter 65.8 J.) \\
Frauen & 104 (durchschn. Alter 65.3 J.) \\
& 265 \\
Die Zahl der Operationen & 174 \\
& 79 \\
radikale & \\
palliative & 12 \\
Op. der schwerwiegenden & \\
Komplikationen &
\end{tabular}

Tabelle 2. Chirurgie des kolorektalen Karzinoms 1989-1998 Tumorlokalisation und Histologie

$\begin{array}{ll}\text { Tumorlokalisation } & \\ \text { Rectum } & 32.4 \% \\ \text { Sigma } & 25.7 \% \\ \text { Rectosigma } & 16.2 \% \\ \text { andere } & 25.7 \%\end{array}$

Histologie $\begin{array}{lr}\text { Adenokarzinom } & 88 \% \\ \text { undifferenziertes Karzinom } & 9 \%\end{array}$ andere $3 \%$

Tabelle 3. Ergebnisse der chirurgischen Therapie des kolorektalen Karzinoms 1989-1998

$$
\begin{array}{lr}
\begin{array}{l}
\text { Die Zahl der Komplikationen } \\
\text { belanglosen (Eitern, Wundendehiscenz usw.) } \\
\text { belangvollen (Dehiscenz der Anastomose usw.) }
\end{array} & 16(6.3 \%) \\
12(4.7 \%)
\end{array}
$$

Tabelle 4. Klassische onkologische Marker mit Spezifität für kolorektales Karzinom

(Laboratorium der onkologischen Klinik der medizinischen Fakultät Olomouc)

$\begin{array}{ll}\text { Benennung } & \text { Norm } \\ \text { Karzinom-embryonaler Antigen (CEA) } & 0-5 \mathrm{ng}(\mathrm{ev} \text {. bis } 10 \mathrm{ng}) \\ \text { Karbohydratenantigen (CA 19-9) } & \text { bis } 37 \mathrm{U} / \mathrm{ml} \\ \text { Gewebe-polypeptidenantigen (TPA) } & \text { bis } 1 \mathrm{ng} / \mathrm{ml}=100 \mathrm{U} / 1 \\ \text { Lipidverbundene Sialsäure (LSA) } & \text { bis } 22 \mathrm{mg} / 100 \mathrm{ml}=220 \mathrm{mg} / \mathrm{l}\end{array}$

Tabelle 5. Übersicht der untersuchten Parameter im Rahmen der Zusammenstellung der prädiktiven Onkologie

(Laboratorium der experimentellen Medizin, Kinderklinik der medizinischen Fakultät und des Fakultätskrankenhauses Olomouc)
I. Chemoresistenz
II. Proliferative Aktivität und genetische Stabilität des Tumors
III. Weitere prognostische Parameter

Tabelle 6. Chemoresistenz

* Untersuchung der Chemoresistenz in vitro - MTT Test 38 standardgetesten Medikamente - TCS 50

* Feststellen der Expression der MDR-assotiierten Proteine P - Glykoprotein (WB, FACS, ICC, IHC),

MRP (WB, ICC, IHC),

LRP (WB, ICC, IHC),

Topoisomerase I (WB, ICC, IHC),

Topoisomerase II (WB, ICC, IHC),

Glutathion-S-Transferase (WB, ICC, IHC)

* Beurteilung der medizinischen Antwort in vivo aus bioptischem Material

Das Prozent der apoptoischen Zellen (Morfologie,TUNEL.DNALadder)

Analyse des Zellenzyclus (FACS, WB, ICC, IHC)

Tabelle 7. Proliferative Aktivität und genetische Stabilität des Tumors

* DNA-Synthese (BrdU Inkorporation, 3H-Thymidin Inkorporation)

* PCNA (WB, ICC, IHC)

* $\mathrm{Ki67}$ (ICC, IHC)

* Zycline (WB, FACS, ICC, IHC)

* Ploidität der Tumorzellen (FACS)

* Analyse des Zellenzyclus (FACS, WB, ICC, IHC)

* Funktion p53 - Proteinkummulation (WB, ICC, IHC)

* Veränderungen in TP53 Lokus-Deletion, Amplifikation (FISH)

Tabelle 8. Weitere prognostische Parameter

* $\quad \mathrm{RB}$ (WB, ICC, IHC, FISH)

* c-myc (SB, WB, ICC, IHC, FISH)

n-myc (WB, ICC, IHC)

* Her2/neu (WB, ICC, IHC, FISH)

* Die Tumorvaskularisation (Morfologie, CD61)

* Das Prozent der apoptoischen Zellen (Morfologie, TUNEL.DNALadder)

* Aktivität der Telomerase (PCR)

* Feststellung der Länge der Telomeren (SB)

* Die Expression und Aktivität der Proteine des Zellenzyclus: cdk 1-7, Zycline A, D, E, B, H, der natürlichen cdk-Inhibitoren: p21, p27, p57, p15, p16 (FACS, WB, ICC, IHC, ELISA, Substratphosphorilation-Teste)

* Aktivität der Matrix-Metaloproteinasen (ICC, IHC, ELISA, Enzymteste)

\section{SCHLUSSFOLGERUNGEN}

Von der begonnenen prospektiven Untersuchungsreihe erwarten wir einen wesentlichen Beitrag für die Diagnostik und Behandlung von Patienten mit kolorektalem Karzinom in unserem Einzugsbereich. Wir erhoffen uns eine Optimierung der adjuvanten Therapie und möglicherweise auch Ansätze für neoadjuvante Therapien. Weiterhin wird in Zukunft über die Erweiterung der Analytik und die Einführung neuer Therapien, z. B. spezifischer Vaccinationen, nachzudenken sein. 


\section{LITERATUR}

1. Arnold, M. W., Young, D. M., Hitchcock, C. L., Barbera-Guillem, E., Nieroda, C., Martin, E. W. jr. (1998) Staging of colorectal cancer: biology vs. morfology. Dis. Colon Rectum, 41, 12, 1482-1487.

2. Baba, S. (1998) Recent advances in molecular genetics of colorectal cancer. World J. Surg., 21, 678-687.

3. Brown, T., Aldous, W., Lance, R., Blaser, J., Baker, T., Williard, W. (1998) The association between telomerase, p53, and clinical staging in colorectal cancer. Am. J. Surg., 175, 364-366.

4. Calaluce, R., Miedema, B. W., Yesus, Y. W. (1998) Micrometastasis in colorectal carcinoma: a review. J. Surg. Oncol., 67, 194-202.

5. Caldas, C. (1997) Molecular staging of cancer: is it time? Lancet, 350, 9073, 231.

6. Chung, D. C. (1998) Molecular prognostic markers and colorectal cancer : the search goes on. Gastroenterology, 114, 1330-1332.

7. Dietel, M. (1998) Molecular pathology of colorectal cancer: from phenotype to genotype. Recent Results Cancer Res., 1946, 3-19.

8. Dutta, S. K., Nair, P. P. (1998) Noninvasive detection of colorectal cancer by molecular tools: comming on age. Gastroenterology, 114, 1333-1335.

9. Fujiwara, T., Tanaka, N. (1998) Molecular surgery for human colorectal cancer with tumor suppresor p53 gene transfer. Nippon Geka Gakkai Zasshi, 99, 463-468.

10. Gryfe, R., Swallow, C., Bapat, B., Redstom, M., Gallinger, S., Couture, J. (1997) Molecular biology of colorectal cancer. Curr. Probl. Cancer, 21, 233-300.

11. Itoh, F., Yamamoto, H., Fukushima, H., Matsuno, K., Imai, K. (1998) Recent advance of prognostic factor in human colorectal cancer. Gan To Kagaku Ryoho, 25, 2028-2035.
12. Jen, J., Johnson, C., Levin, B. (1998) Molecular approaches for colorectal cancer screening. Eur. J. Gastroenterol. Hepatol., 10, 213-217.

13. Jessup, J. M., Loda, M., Bleday, R. (1998) Clinical and molecular prognostic factor in sphincter-preserving surgery for rectal cancer. Semin. Radiat. Oncol., 8, 1, 54-69.

14. Johnson, P. G., Allegra, C. J. (1995) Colorectal cancer biology: clinical implication. Semin. Oncol., 22, 5, 418-432.

15. Laurent-Puig, P., Cugnenc, P. H. (1997) The application of molecular technics in the management of colorectal cancer. J. Chir. (Paris), 134, 279-282.

16. Lynch, H. T., Lemon, S. J., Karr, B., Franklin, B., Lynch, J. F., Watson, P., Tinkley, S., Lerman, C., Carter, C. (1997) Etiology, natural history, management and molecular genetics of hereditary nonpolyposis colorectal cancer (Lynch syndromes): genetic counseling implications. Cancer Epidemiol. Biomarkers Prev., 6, 987-991.

17. Muto, T. (1998) Colorectal carcinogenesis: a historical review. Nippon Geka Gakkai Zasshi, 99, 351-356.

18. Schackert, H. K., Hahn, M., Pistorius, St., Saeger, H. D. (1999) Präventive Chirurgie des vererbten colorectalen Carcinoms als Folge molekularer Diagnostik. Chirurg., 70, 345-352.

19. Smyth, J. P. (1996) Cancer genetics and cells and molecular biology. Is this the way forward? Chest, 109 , 5suppl, $125 \mathrm{~S}-129 \mathrm{~S}$.

20. Spataro, V. (1998) Recent advances in the molecular genetics of cancer second joint conference of the American Association of Cancer Research and the European Association of Cancer Research. Ann. Oncol., 9, 1, 23-29.

21. Tomlinson, I., Ilyas, M., Novelli, M. (1997) Molecular genetics of colon cancer. Cancer Metastasis Rev., 16, 1-2, 67-79. 